\title{
Estudio arqueométrico de figurillas cerámicas mayas de Calakmul (Campeche, México)
}

\author{
M. GARCÍA-HERAS ${ }^{1-2}$, J. REYES TRUJEQUE ${ }^{3}$, R. RUIZ GUZMÁN ${ }^{4}$, M.A. AVILÉS ESCAÑO ${ }^{5}$, \\ A. RUIZ CONDE ${ }^{5}$ Y P.J. SÁNCHEZ SOTO ${ }^{5}$ \\ ${ }^{1}$ Centro Nacional de Investigaciones Metalúrgicas, CENIM-CSIC. Avda. Gregorio del Amo, 8. 28040 Madrid. \\ ${ }^{2}$ Instituto de Historia, IH-CSIC. Serrano, 13. 28001 Madrid. \\ ${ }^{3}$ Universidad Autónoma de Campeche. Programa de Corrosión del Golfo de México. Avda. Agustín Melgar, s/n. 24013 Campeche, México. \\ ${ }^{4}$ Universidad Autónoma de Campeche. Centro de Investigaciones Históricas y Sociales. Avda. Agustín Melgar, s/n. 24013 Campeche, México. \\ ${ }^{5}$ Instituto de Ciencia de Materiales, Centro Mixto CSIC-Universidad de Sevilla. Americo Vespucio, 49.41092 Isla de la Cartuja, Sevilla.
}

\begin{abstract}
En este trabajo se presentan y discuten los resultados de la caracterización arqueométrica realizada sobre un conjunto representativo de figurillas cerámicas del Clásico Tardío procedentes de las excavaciones arqueológicas efectuadas en la ciudad maya de Calakmul (Campeche, México). Los objetivos principales han sido recabar información sobre la tecnología de manufactura de estas cerámicas y evaluar su posible procedencia. La caracterización arqueométrica se ha llevado a cabo mediante las siguientes técnicas complementarias: microscopía óptica convencional (MO), difracción de rayos X (DRX) de polvo, espectroscopía infrarroja por Transformada de Fourier (FT-IR) y fluorescencia de rayos X (FRX). Con el fin de aislar grupos cerámicos con un perfil químico similar, los datos obtenidos con FRX se han analizado también con técnicas exploratorias de análisis estadístico multivariante. Los resultados indican que la mayoría de las figurillas se manufacturaron localmente con una materia prima arcillosa rica en carbonato de calcio (calcita). También se ha identificado un pequeño grupo de cerámicas rico en carbonatos mixtos de calcio y magnesio (dolomita), que podría ser foráneo o procedente de otra zona de la región de Calakmul. Estos datos sugieren que la producción de figurillas cerámicas pudo estar asociada a talleres ligados al monopolio sobre la producción cerámica que el poder centralizado de la ciudad de Calakmul ejerció en este período.
\end{abstract}

Palabras clave: Cerámica maya; Figurillas; Clásico Tardio; Calakmul; Arqueometría.

Archaeometric study of Mayan ceramic figurines from Calakmul (Campeche, México).

The results derived from an archaeometric characterization study undertaken on a representative ensemble of Late Classic ceramic figurines coming from the archaeological field work carried out in the Mayan town of Calakmul (Campeche, México) are reported and discussed in this paper. The main goals of the research were to gather some information on the technology of manufacture of these ceramics and provide some insights into their probable provenance. The archaeometric characterization was accomplished through the following complementary techniques: conventional optical microscopy (OM), X-ray powder diffraction (XRD), Fourier transform infrared spectroscopy (FT-IR) and X-ray fluorescence (XRF). In order to isolate ceramic groups with a similar chemical profile, XRF data were also analyzed by exploratory multivariate statistical techniques. The resulting data indicate that most of the figurines were locally manufactured from a calcium carbonate (calcite)-rich clay raw material. A small ceramic group containing mixed calcium and magnesium carbonates (dolomite), which could be foreign or from other area of the Calakmul region, was also identified. These data suggest that the production of ceramic figurines could be associated to workshops linked to the monopoly on ceramic production performed by the centralized power of the town of Calakmul during this period.

Keywords: Mayan ceramics; Figurines; Late Classic; Calakmul; Archaeometry.

\section{INTRODUCCIÓN Y OBJETIVOS}

La ciudad de Calakmul (Campeche, México) fue uno de los centros geopolíticos y económicos más importantes de la región maya central del Petén entre el Preclásico Tardío ( 200 a.C.) y el Clásico Tardío ( 800 d.C.) (Figura 1). La importancia de este centro se pone de manifiesto en su desarrollo urbano con la edificación de conjuntos palaciegos que conforman una gran acrópolis en torno a plazas y patios, así como la construcción de dos grandes pirámides que dan nombre a la ciudad: Ca (dos)-lak (adyacente)-mul (montículo o pirámide) “Dos montículos adyacentes" $(1,2)$.
La zona central de la antigua ciudad maya de Calakmul está constituida por un área conocida como la Gran Plaza, un eje que vertebraba en gran parte la vida pública de la ciudad. En el lado sur de este espacio se localiza la llamada Estructura II (Figura 1), que se corresponde con una de las dos pirámides erigidas en esta ciudad. Su construcción se inició en el Preclásico Tardío y consta de un basamento escalonado de esquinas remetidas, coronado por edificios decorados con estuco rojo, y una escalera central exenta. La Estructura II puede ser considerada la construcción más sobresaliente de 


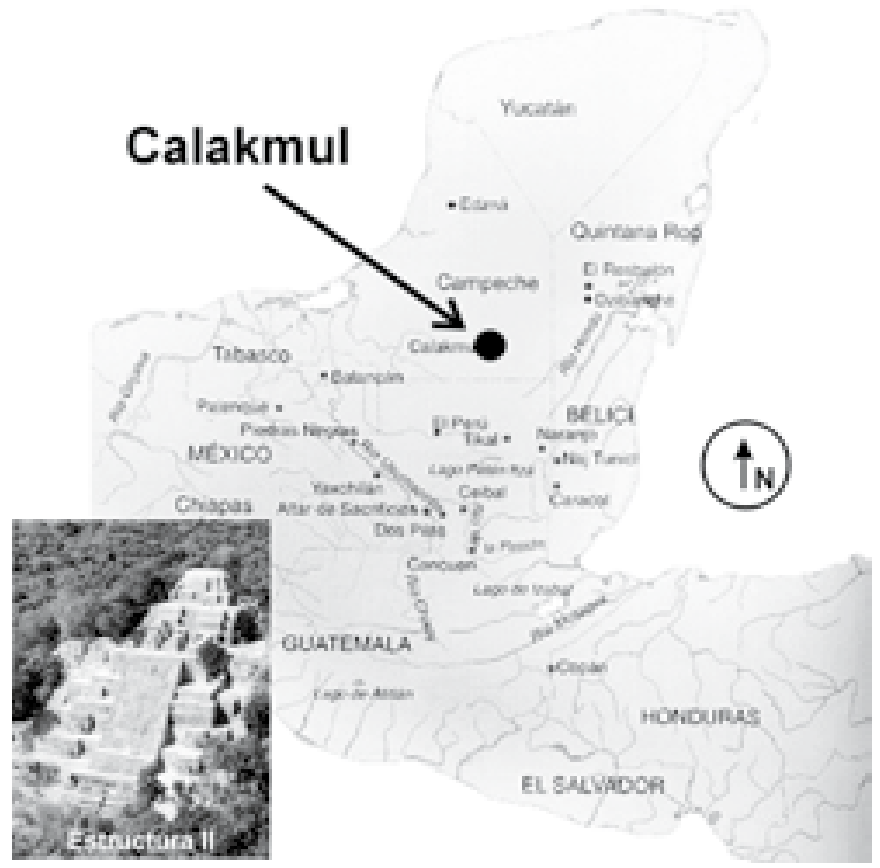

Fig. 1- Situación geográfica y Estructura II de la ciudad maya de Calakmul (Campeche, México). Fotografía de la Estructura II: Carlos Vidal Anglés.

Calakmul tanto por su complejidad arquitectónica como por su gran volumen. De hecho, y a pesar del derrumbe de la parte superior del edificio, se encuentra actualmente, con sus $45 \mathrm{~m}$ de altura, entre las mayores construcciones del área cultural maya (3).

Los trabajos arqueológicos emprendidos en esta ciudad a lo largo de las últimas décadas han proporcionado un extraordinario conjunto de hallazgos relacionados con las comunidades mayas que habitaron el asentamiento entre los períodos Preclásico Tardío y Clásico Terminal. Entre los distintos materiales que conforman este conjunto de hallazgos destaca, por su variedad y riqueza, una importante colección de cerámicas y, en particular, aquellas recuperadas en las distintas campañas de excavación arqueológica llevadas a cabo en la Estructura II. De la totalidad de ejemplares cerámicos de los que se dispone, un número importante está constituido por figurillas (zoomorfas y antropomorfas) e instrumentos musicales como ocarinas, realizadas con técnicas de modelado y moldeado. Alrededor de un $80 \%$ de las figurillas fueron localizadas en la propia Estructura II, situándose en un porcentaje cercano al $90 \%$ las que pertenecen al período cronológico del Clásico Tardío (600-900 d.C.), según sus características tipológicas y estilísticas (4). Las figurillas de Calakmul representan manifestaciones de carácter político, social y religioso, además de rasgos culturales como la propia vestimenta (5).

Teniendo en cuenta la importancia de estos materiales cerámicos, no sólo para el estudio de la cultura maya en el período comprendido entre el Preclásico Tardío y el Clásico Terminal en la región central del Petén, sino también para el conocimiento científico de la evolución de la tecnología cerámica en las culturas prehispánicas de Mesoamérica, en este trabajo se ha caracterizado una muestra representativa de figurillas cerámicas pertenecientes al Clásico Tardío mediante distintas técnicas de análisis fisicoquímico, como es habitual en estudios arqueométricos $(6,7)$, con el propósito de estudiar su tecnología de producción y estimar si se trata de producciones locales o foráneas al área de la propia ciudad de Calakmul.

\section{PARTE EXPERIMENTAL}

\subsection{Muestras estudiadas}

En este estudio arqueométrico se analizaron un total de 17 muestras cerámicas pertenecientes a 13 figurillas y cuatro instrumentos musicales (tres tipificados como ocarinas y uno como una flauta) (4). Todas ellas procedían de la fachada principal de la Estructura II de la ciudad maya de Calakmul (Figura 1). En la Tabla I se ofrece su descripción y su ubicación espacial dentro de dicha Estructura. La selección de estas muestras se realizó siguiendo criterios arqueológicos, de modo que estuvieran representados la mayor parte de los niveles y espacios funcionales de la pirámide. En las fotografías de la Figura 2 se muestran algunas de las piezas cerámicas analizadas.

TABLA I. MUESTRAS ANALIZADAS DEL PERÍODO CLÁSICO TARDÍO (600-900 d.C.) DE LA ESTRUCTURA II, FACHADA PRINCIPAL, DE LA CIUDAD MAYA DE CALAKMUL.

\begin{tabular}{|c|c|c|c|}
\hline $\mathrm{N}^{\text {}}$ & MUESTRA & NIVEL & DESCRIPCIÓN \\
\hline 1 & M-3 & 9 & Torso antropomorfo \\
\hline 2 & M-4 & 9 & $\begin{array}{c}\text { Cabeza antropomorfa } \\
\text { con base anular }\end{array}$ \\
\hline 3 & M-5 & 9 & Cabeza zoomorfa \\
\hline 4 & M-7 & 9 & Símbolo de deidad (¿dios K?) \\
\hline 5 & M-8 & 8 & Ocarina \\
\hline 6 & M-14 & 8 & Torso antropomorfo \\
\hline 7 & M-16 & 8 & Ocarina zoomorfa (¿perro?) \\
\hline 8 & M-9 & 7 & Cuerpo antropomorfo \\
con restos de pigmento azul \\
\hline 9 & M-10 & 7 & Fragmento anular \\
\hline 10 & M-17 & 7 & Flauta con cabeza de simio \\
\hline 11 & M-41 & 6 & Ocarina zoomorfa \\
\hline 12 & M-42 & 6 & Sin forma reconocible \\
\hline 13 & M-44 & 6 & Sin forma reconocible \\
\hline 14 & M-45 & 6 & Cabeza con sombrero \\
\hline 15 & M-11 & 5 & Torso antropomorfo \\
\hline 16 & M-12 & 5 & Torso antropomorfo \\
\hline 17 & M-49 & 3 & Cabeza con tocado \\
\hline
\end{tabular}

\subsection{Técnicas de análisis utilizadas}

Todas las muestras seleccionadas se caracterizaron con las siguientes técnicas complementarias: microscopía óptica convencional (MO), difracción de rayos X(DRX), espectroscopía infrarroja por Transformada de Fourier (FT-IR) y fluorescencia de rayos $\mathrm{X}$ (FRX). Los análisis mediante DRX, FT-IR y FRX fueron realizados sobre muestra en polvo, obtenida con un taladro de tungsteno, que fue convenientemente triturada en mortero de ágata hasta lograr un tamaño de grano inferior a $63 \mu \mathrm{m}$, secándose a continuación en estufa a $110{ }^{\circ} \mathrm{C}$ durante $24 \mathrm{~h}$. Seguidamente se envasaron en viales de polietileno convenientemente sellados. En todo caso, se procuró operar siempre con la menor cantidad posible de material (entre 0,5 y 2 g), con el fin de no incidir en exceso en el aspecto original que presentaban estas piezas cerámicas que en su mayoría se encontraban muy bien conservadas. 

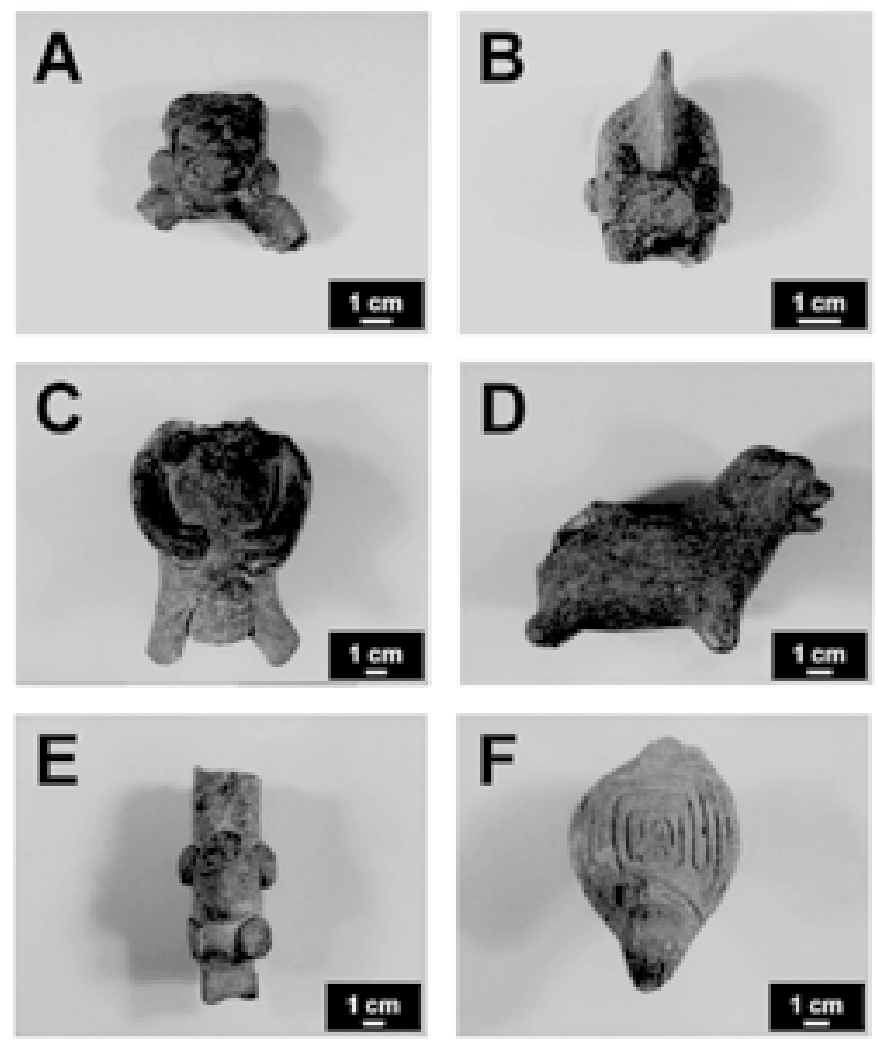

Fig. 2- Fotografías de algunas de las piezas cerámicas analizadas. A) Muestra M-4. Cabeza antropomorfa con base anular. B) Muestra M-5. Cabeza zoomorfa. C) Muestra M-12. Torso antropomorfo. D) Muestra M-16. Ocarina zoomorfa. E) Muestra M-17. Flauta con cabeza de simio. F) Muestra M-41. Ocarina zoomorfa.

El microscopio óptico empleado fue un equipo Nikon Optiphot sobre fracturas cerámicas frescas (8). Para el análisis cualitativo de las fases cristalinas presentes en las muestras estudiadas se empleó la técnica de DRX, siguiendo el método convencional de polvo cristalino y difracción normal utilizando unos $0,2 \mathrm{~g}$ de muestra. Se empleó un difractómetro de rayos $\mathrm{X}$ Siemens modelo D-501, utilizando la radiación $K(\alpha)$ del cobre $(1,5406 \AA ̊ \AA)$, filtro de níquel y monocromador de grafito, a 36 $\mathrm{kV}$ y $26 \mathrm{~mA}$, con una sensibilidad adecuada en el detector, velocidad de goniómetro y registro. Los difractogramas se obtuvieron entre $2 \theta=2-60^{\circ}$. Con objeto de conseguir una mejor identificación de las fases minerales presentes, se empleó un programa de búsqueda automática de fases, bajo condiciones impuestas por el usuario. El sistema empleado fue SICOMP PC 16-20 e impresora gráfica con código de colores para cada fase identificada, utilizando el fichero JCPDS (Joint Committee for Powder Diffraction Standards).

Los espectros FT-IR se realizaron con un equipo Nicolet FTIR en el rango comprendido entre $4000-400 \mathrm{~cm}^{-1}$, llevándose a cabo un número de 32 barridos para cada espectro. No obstante, como la mayoría de las bandas de absorción características de las fases presentes en las muestras analizadas se obtuvieron a un número de onda inferior a $1600 \mathrm{~cm}^{-1}$ (9), sólo aquella región del espectro por debajo de este valor se ha representado en la figura correspondiente. Los análisis se efectuaron preparando pastillas por prensado a $200 \mathrm{MPa}$, con la muestra original mezclada con bromuro potásico.

En el análisis químico mediante FRX se utilizó un espectrómetro secuencial de dispersión de longitudes de onda
Siemens SRS-3000, equipado con cristales analizadores LiF 100, LiF 110, PET, OVO55, OVO120L y ánodo de rodio. Para realizar este análisis, se prepararon pastillas por prensado a $1250 \mathrm{MPa}$ de las muestras originales (alrededor de 0,03 g), en una matriz metálica adecuada, a las que se les adicionó una sustancia orgánica (cera, en torno a 0,45 g) sobre una base de ácido bórico (99,8 \% de pureza). A través de esta técnica se determinaron las concentraciones de los 21 elementos químicos siguientes: 10 de ellos mayoritarios expresados como óxidos en $\%$ en peso $\left(\mathrm{Na}_{2} \mathrm{O}, \mathrm{MgO}, \mathrm{Al}_{2} \mathrm{O}_{3^{\prime}}, \mathrm{SiO}_{2^{\prime}}, \mathrm{P}_{2} \mathrm{O}_{5^{\prime}}, \mathrm{K} 2 \mathrm{O}, \mathrm{CaO}\right.$, $\mathrm{TiO}_{2}, \mathrm{MnO}$ y $\mathrm{Fe}_{2} \mathrm{O}_{3}$ ); y 11 minoritarios y trazas expresados en ppm (V, Cr, Co, Ni, Cu, Zn, Rb, Sr, Y, Zr y Ba).

\subsection{Estudio estadístico}

Con el fin de aislar y estimar la validez estadística de aquellos grupos que mostraran un perfil químico similar, los datos de composición química obtenidos con FRX se analizaron con tres técnicas exploratorias de análisis estadístico multivariante: análisis cluster, análisis de componentes principales y análisis canónico discriminante que incluyó el cálculo de distancias de Mahalanobis (10), utilizando el paquete estadístico Systat v. 10.0 (SPSS Inc., Chicago). Las concentraciones se transformaron en valores logarítmicos para compensar las diferencias de magnitud entre los elementos mayoritarios, minoritarios y trazas en el cálculo de los coeficientes de similaridad (11). En los análisis estadísticos no se tuvieron en cuenta las concentraciones correspondientes a los elementos $\mathrm{V}, \mathrm{Cr}, \mathrm{Co}, \mathrm{Ni}, \mathrm{Rb}$, Sr e $\mathrm{Y}$, debido a que en algunas muestras no fue posible su determinación al estar sus concentraciones próximas a los límites de detección del equipo. Asimismo, tampoco se utilizaron las concentraciones de $\mathrm{P}_{2} \mathrm{O}_{5}$ por ser un óxido muy sensible a los cambios postdeposicionales experimentados por las cerámicas arqueológicas durante su largo enterramiento, como ha sido ampliamente demostrado en la bibliografía al uso (12). Por consiguiente, el análisis estadístico de los datos de FRX se llevó a cabo utilizando sólo las 14 variables siguientes: $\mathrm{Na}_{2} \mathrm{O}, \mathrm{MgO}, \mathrm{Al}_{2} \mathrm{O}_{3^{\prime}}, \mathrm{SiO}_{2}, \mathrm{~K}_{2} \mathrm{O}, \mathrm{CaO}$, $\mathrm{TiO}_{2}, \mathrm{MnO}, \mathrm{Fe}_{2} \mathrm{O}_{3^{\prime}} \mathrm{Cu}, \mathrm{Zn}, \mathrm{Sr}, \mathrm{Zr}$ y Ba.

\section{RESULTADOS Y DISCUSIÓN}

\subsection{Microscopía óptica}

La observación de las muestras mediante MO, determinó que se trataba de una cerámica con una macroporosidad abundante, de coloración homogénea predominantemente ocre. En la matriz arcillosa se observó la presencia de numerosas inclusiones no plásticas de color blanquecino y grisáceo, con bordes mayoritariamente redondeados y un amplio rango de tamaños que alcazaban, en algunos casos, hasta 300-400 $\mu \mathrm{m}$. Tanto el tamaño como la morfología redondeada de estas inclusiones sugieren que ya se hallaban en el sedimento arcilloso de forma natural y que, con toda probabilidad, no fueron añadidas como desgrasante de forma intencionada por los alfareros mayas (Figura 3). Por lo demás, no se hallaron diferencias destacables entre las 17 muestras analizadas.

\subsection{Difracción de rayos $X$}

El análisis cualitativo de las fases cristalinas mediante DRX indica la existencia de tres grupos mineralógicos distintos en las muestras cerámicas estudiadas. Un primer grupo 

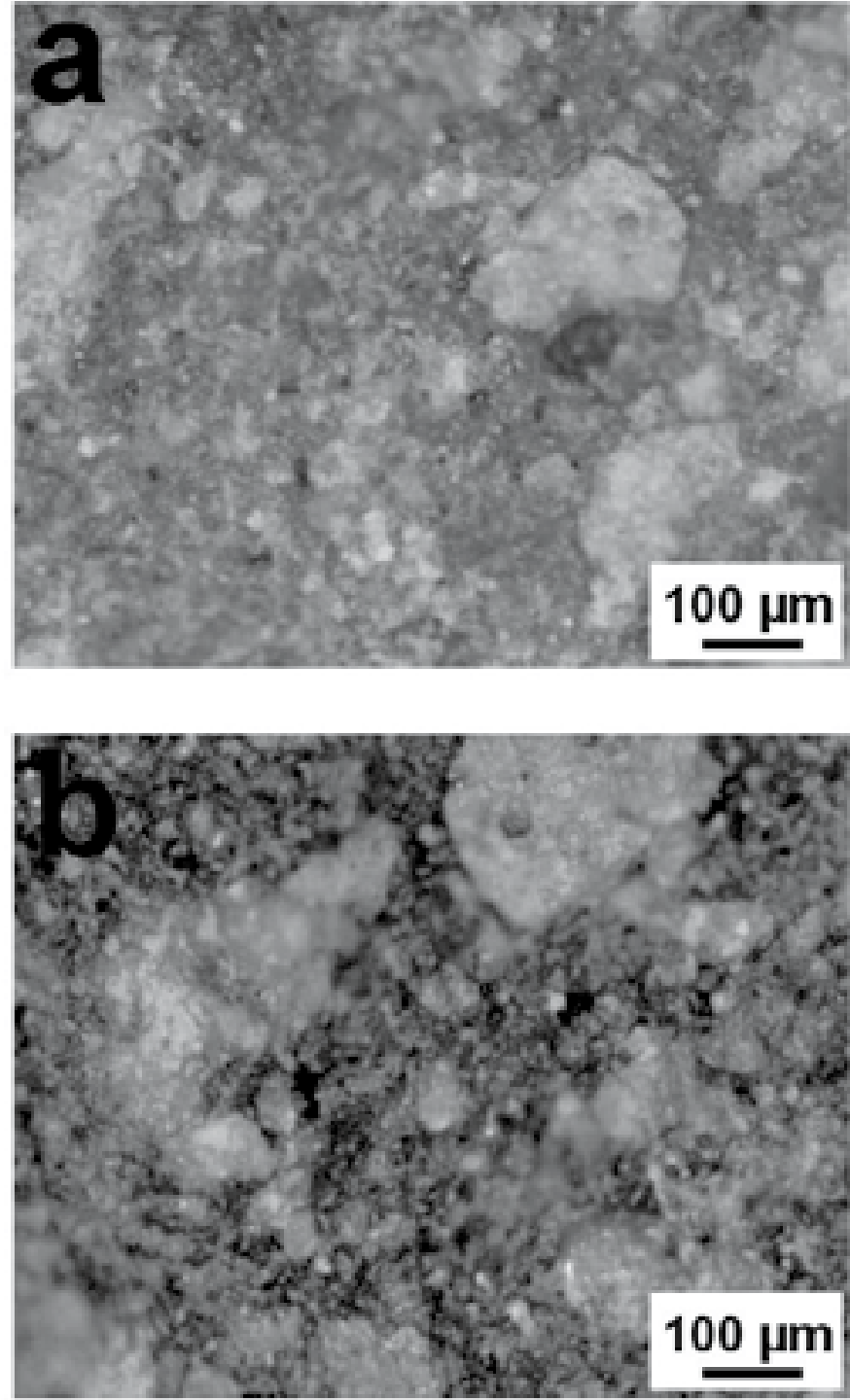

Fig. 3- Imágenes de MO de algunas de las piezas cerámicas analizadas. Fractura fresca. a) Muestra M-9. b) Muestra M-16.

con dolomita $\left[\mathrm{CaMg}\left(\mathrm{CO}_{3}\right)_{2}\right]$ como fase predominante, un segundo grupo en el que la calcita $\left(\mathrm{CaCO}_{3}\right)$ es su fase mayoritaria y, finalmente, un tercer grupo en donde sólo se identifica una escasa presencia de cuarzo $\left(\mathrm{SiO}_{2}\right)$ y calcita sobre un fondo mayoritariamente amorfo. En ninguno de los tres grupos se detectan fases de neoformación inducidas por reacciones térmicas durante la cocción. En la Figura 4 se ofrecen los difractogramas más significativos de los tres grupos mencionados.

El grupo con dolomita como fase predominante está compuesto por cuatro muestras (M-5, M-8, M-9 y M-41). Junto a la dolomita también se detectó una pequeña cantidad de calcita. En todas las muestras de este grupo el cuarzo aparece en muy baja concentración, excepto en la muestra M-5 en la que sus picos característicos fueron más intensos, o en la muestra M-8 en donde apenas se detectó. En esta agrupación la presencia de mica y otros filosilicatos sin alterar por efecto de la temperatura de cocción fue muy escasa. Por otro lado, en el grupo con calcita como fase mayoritaria, que está compuesto por 11 muestras (M-3, M-4, M-7, M-10, M11, M-12, M-14, M-16, M-17, M-42 y M-49), la presencia de
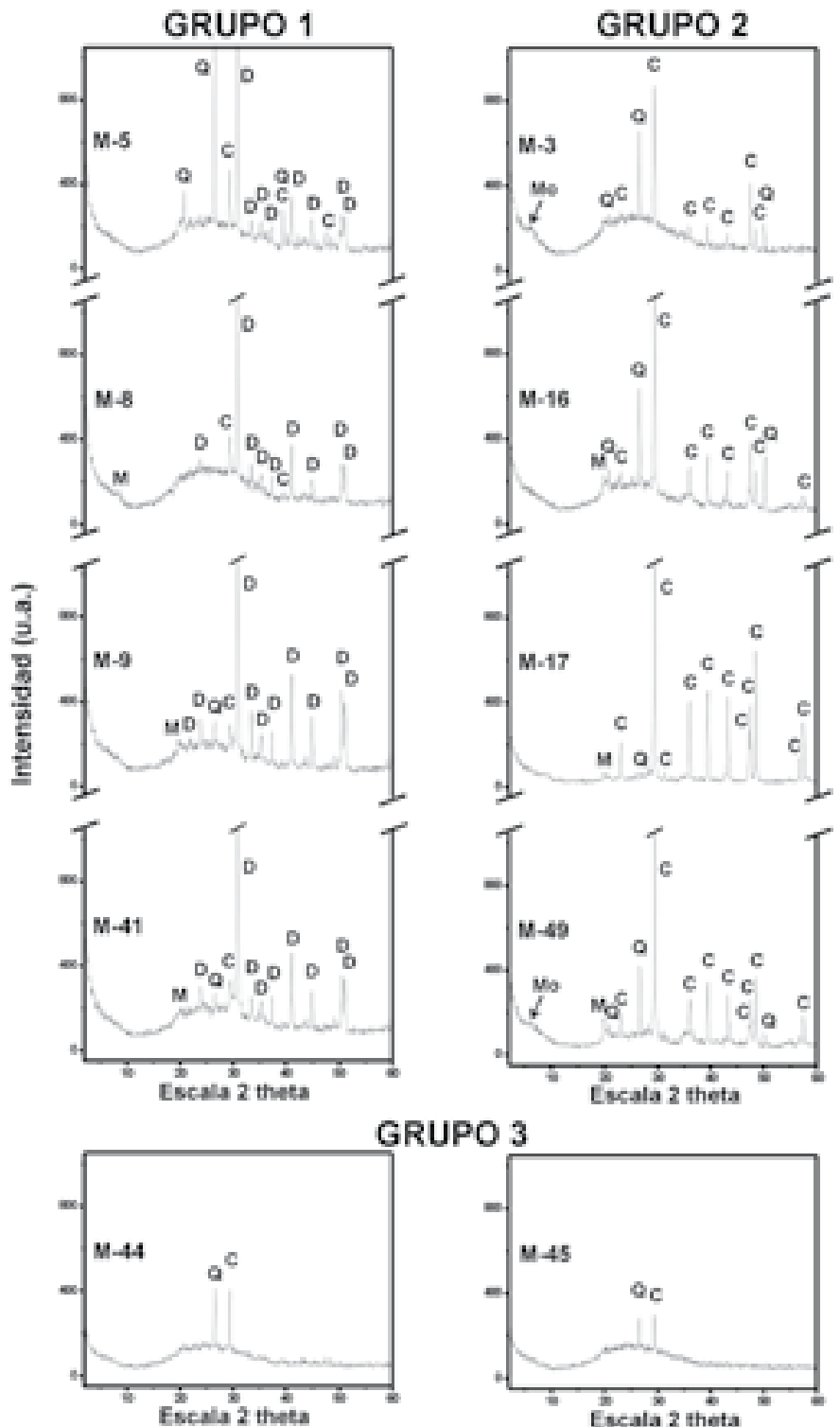

GRUPO 3

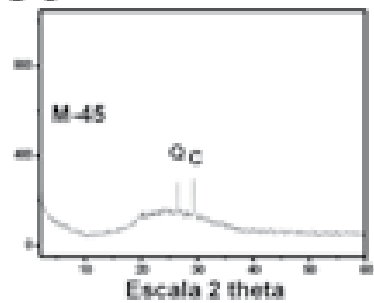

Fig. 4- Difractogramas de rayos $X$ de algunas muestras representativas. C Calcita. D Dolomita. M Mica y otros filosilicatos. Mo Posible montmorillonita. Q Cuarzo.

cuarzo es, en general, más acusada que en el grupo anterior, aunque la mica y otros filosilicatos es igualmente muy escasa. Sólo en las muestras M-3 y M-49 se detectaron reflexiones de pequeña intensidad que podrían corresponderse con clorita o montmorillonita (Figura 4), seguramente en su fase deshidroxilada todavía no transformada por la cocción. De todas formas, para poder identificar correctamente uno $\mathrm{u}$ otro tipo de arcilla sería necesario poder disponer en el futuro de muestras de arcillas de la región de Calakmul en las que poder llevar a cabo agregados orientados y distintos ensayos térmicos. Por lo demás, en ninguna de las 11 muestras de este grupo se detectó la presencia de dolomita. Finalmente, el tercer grupo, compuesto sólo por las muestras M-44 y M-45, muy similares entre sí, presenta una muy escasa presencia de cuarzo y calcita sobre una banda ancha localizada entre 20 y $30^{\circ}(2 \theta)$ que podría corresponderse con sílice vítrea (Figura 4). Los difractogramas de estas dos muestras presentan, en cualquier caso, unas reflexiones de muy escasa intensidad, 
lo que además de diferenciarlas claramente del resto de las muestras podría indicar que se trata de cerámicas sometidas a una temperatura de cocción más elevada que las pertenecientes a las otras dos agrupaciones.

\subsection{Espectroscopía infrarroja}

Los espectros de infrarrojo corroboran los resultados obtenidos mediante DRX, ya que también pueden identificarse, a partir de las fases mineralógicas detectadas, tres grupos distintos. La Figura 5 ofrece los espectros más significativos registrados en las muestras estudiadas. Al igual que en los análisis por DRX, en ninguno de los tres grupos se detectan fases de neoformación inducidas por reacciones térmicas durante la cocción.
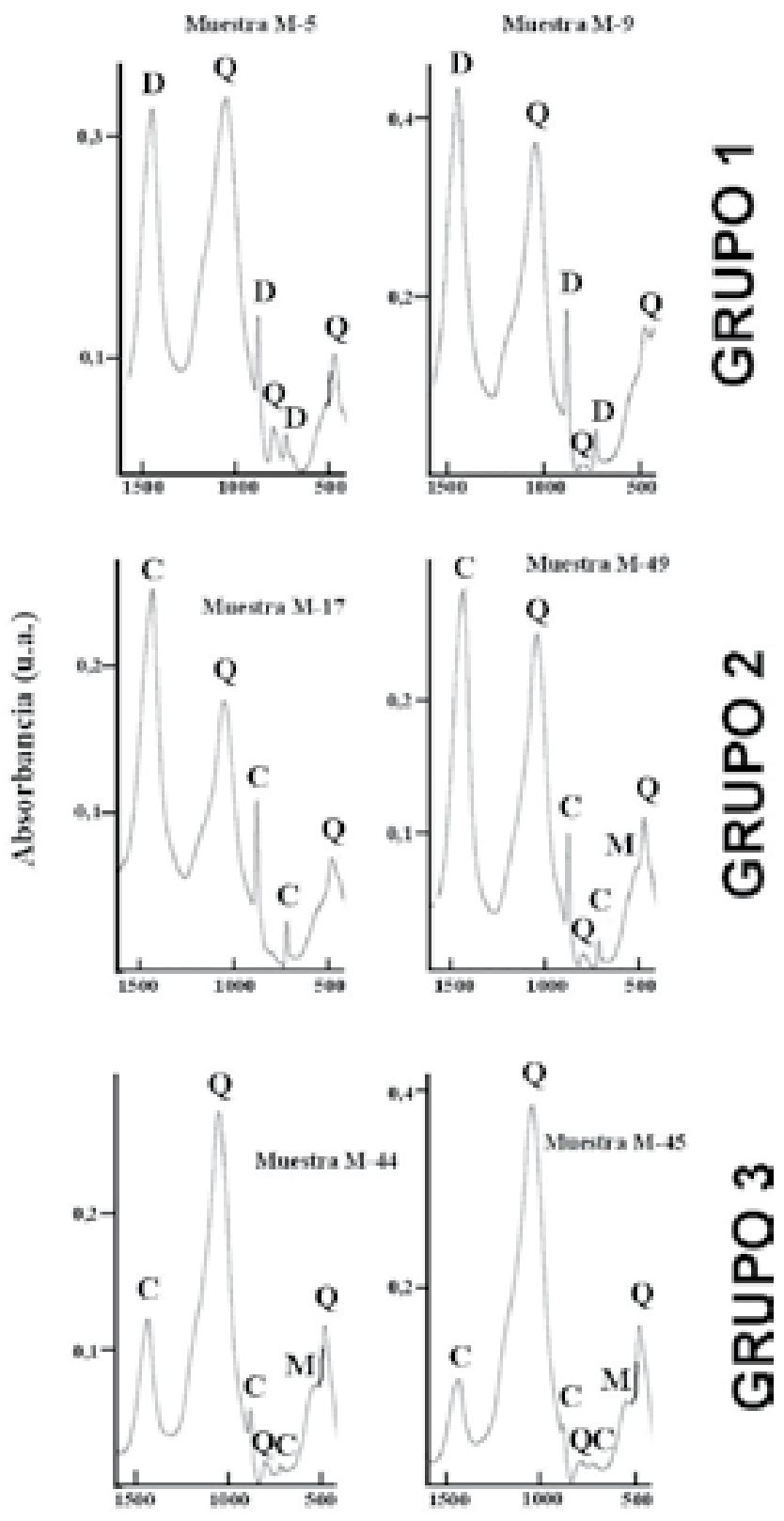

Nümcros de onda $\left(\mathrm{cm}^{3}\right)$

Fig. 5- Espectros FT-IR de algunas muestras representativas. C Calcita. D Dolomita. M Mica y otros filosilicatos. Q Cuarzo.
En el primer grupo, caracterizado por la presencia de dolomita como fase predominante, ésta se identifica bien a partir de su banda principal de absorción en torno a los 1445 $\mathrm{cm}^{-1} \mathrm{y}$ a las bandas secundarias situadas alrededor de $880 \mathrm{y}$ $728 \mathrm{~cm}^{-1}$, respectivamente. Al igual que en los difractogramas, todas las muestras de este grupo, excepto la M-5, presentan una mayor intensidad, tanto en la banda principal de absorción como en las secundarias, de la dolomita con respecto al cuarzo, cuyas bandas se sitúan en torno a los 1050, 797 y 472 $\mathrm{cm}^{-1}$. Por el contrario, en los espectros correspondientes a esta agrupación resulta difícil identificar la presencia de calcita, como se detectaba a través de DRX, ya que sus bandas de absorción características, situadas alrededor de 1420, 877 y 714 $\mathrm{cm}^{-1}$ respectivamente, podrían quedar encubiertas o solapadas por la mayor intensidad de las bandas correspondientes a la dolomita. De igual forma, tampoco se detectan las bandas de la mica y de otros filosilicatos (9).

En el segundo de los grupos, que contiene calcita como fase mayoritaria, ésta se determina a partir de su banda principal de absorción en torno a los $1420 \mathrm{~cm}^{-1}$ y de las transiciones situadas a 877 y $714 \mathrm{~cm}^{-1}$, respectivamente. Las bandas de absorción de la calcita presentan siempre una mayor intensidad que las correspondientes al cuarzo. Sólo en algunas muestras se puede determinar la presencia de mica a partir de la transición situada alrededor de $550 \mathrm{~cm}^{-1}$ $(9,13)$, que es la única banda de absorción característica de la mica que no queda solapada en los espectros por las bandas de la calcita y del cuarzo. En las muestras M-3 y M-49 no se detecta la posible presencia de clorita o montmorillonita como indicaban los análisis mediante DRX. Tampoco se detecta dolomita en ninguna de las 11 muestras de esta agrupación. Por último, el tercer grupo, compuesto sólo por dos muestras y caracterizado por la presencia de cuarzo y calcita como fases predominantes, muestra alguna diferencia respecto a los análisis por DRX, debido a que en los espectros registrados la banda de absorción principal del cuarzo, situada en torno a los $1050 \mathrm{~cm}^{-1}$ y las transiciones a 797 y $472 \mathrm{~cm}^{-1}$ respectivamente, son siempre de mayor intensidad que las asociadas a la calcita. Por lo tanto, el cuarzo se muestra como fase predominante frente a la calcita. Asimismo, a través de los espectros de infrarrojo y a diferencia de la DRX, también se determina en este último grupo la presencia de mica a partir de la transición situada alrededor de $550 \mathrm{~cm}^{-1}$. Como ocurre en las muestras adscritas al segundo de los grupos, se trata de la única banda de absorción característica de la mica que no queda solapada en los espectros por las bandas del cuarzo y de la calcita. Además, la presencia de mica sugiere que, aunque las cerámicas de este tercer grupo fueron sometidas a una temperatura de cocción más elevada, ésta no fue suficiente para destruir por completo la mica.

\subsection{Fluorescencia de rayos $X$ y estudio estadístico}

Los resultados de los análisis químicos obtenidos mediante FRX en las 17 muestras cerámicas se ofrecen en la Tabla II. Una vez transformados en valores logarítmicos se sometieron a un análisis estadístico exploratorio mediante análisis cluster y análisis de componentes principales, con el fin de llevar a cabo una primera aproximación a la estructura que presentaban los datos y determinar cuáles eran las variables que mostraran un mayor poder discriminante en la separación de aquellos grupos cerámicos con un perfil químico similar (10). Estos análisis numéricos previos, no ofrecidos en este trabajo por razones de espacio, indicaron que ocho de las 14 variables 
TABLA II. RESULTADOS DE LOS ANÁLISIS QUÍMICOS MEDIANTE FRX

\begin{tabular}{|c|c|c|c|c|c|c|c|c|c|c|c|c|c|c|}
\hline & $\begin{array}{c}\mathrm{Na}_{2} \mathrm{O} \\
(\%)\end{array}$ & $\begin{array}{c}\mathrm{MgO} \\
(\%)\end{array}$ & $\begin{array}{c}\mathrm{Al}_{2} \mathrm{O}_{3} \\
(\%)\end{array}$ & $\begin{array}{c}\mathrm{SiO}_{2} \\
(\%)\end{array}$ & $\begin{array}{l}\mathrm{K}_{2} \mathrm{O} \\
(\%)\end{array}$ & $\begin{array}{c}\mathrm{CaO} \\
(\%)\end{array}$ & $\begin{array}{l}\mathrm{TiO}_{2} \\
(\%)\end{array}$ & $\begin{array}{c}\mathrm{MnO} \\
(\%)\end{array}$ & $\begin{array}{c}\mathrm{Fe}_{2} \mathrm{O}_{3} \\
(\%)\end{array}$ & $\begin{array}{c}\mathrm{Cu} \\
(\mathrm{ppm})\end{array}$ & $\begin{array}{c}\mathrm{Zn} \\
(\mathrm{ppm})\end{array}$ & $\begin{array}{c}\mathrm{Sr} \\
(\mathrm{ppm})\end{array}$ & $\begin{array}{c}\mathrm{Zr} \\
(\mathrm{ppm})\end{array}$ & $\begin{array}{c}\mathrm{Ba} \\
(\mathrm{ppm})\end{array}$ \\
\hline M-3 & 0,30 & 1,76 & 20,35 & 49,73 & 1,00 & 20,05 & 0,76 & 0,76 & 5,05 & 60 & 81 & 470 & 170 & 1591 \\
\hline M-4 & 0,35 & 3,27 & 13,99 & 53,96 & 0,71 & 23,17 & 0,44 & 0,06 & 3,79 & 75 & 137 & 557 & 166 & 1641 \\
\hline M-5 & 0,31 & 8,44 & 14,58 & 50,36 & 1,30 & 20,11 & 0,79 & 0,04 & 3,80 & 234 & 206 & 417 & 243 & 1583 \\
\hline M-7 & 0,35 & 3,27 & 13,99 & 53,96 & 0,71 & 23,17 & 0,44 & 0,06 & 3,79 & 75 & 137 & 557 & 166 & 1641 \\
\hline M-8 & 0,31 & 12,71 & 10,01 & 48,06 & 1,20 & 25,03 & 0,29 & 0,04 & 2,18 & 45 & 76 & 285 & 80 & 1219 \\
\hline M-9 & 0,38 & 13,18 & 9,88 & 50,91 & 1,40 & 21,75 & 0,30 & 0,04 & 2,04 & 41 & 93 & 462 & 98 & 489 \\
\hline M-10 & 0,31 & 5,47 & 8,95 & 51,29 & 1,81 & 28,36 & 0,29 & 0,05 & 3,30 & 46 & 94 & 760 & 120 & 715 \\
\hline M-11 & 0,26 & 5,53 & 9,10 & 53,00 & 1,90 & 25,91 & 0,32 & 0,05 & 3,74 & 41 & 88 & 573 & 95 & 1100 \\
\hline M-12 & 0,32 & 2,45 & 17,95 & 53,84 & 1,10 & 19,54 & 0,52 & 0,33 & 3,63 & 59 & 107 & 775 & 229 & 1983 \\
\hline M-14 & 0,66 & 2,01 & 20,17 & 51,39 & 1,28 & 19,67 & 0,64 & 0,07 & 3,82 & 156 & 160 & 487 & 159 & 1890 \\
\hline M-16 & 0,46 & 2,77 & 19,97 & 54,91 & 0,93 & 15,58 & 0,68 & 0,12 & 4,17 & 49 & 111 & 733 & 248 & 2914 \\
\hline M-17 & 0,35 & 3,07 & 7,25 & 42,27 & 0,84 & 44,28 & 0,15 & 0,02 & 1,35 & 44 & 76 & 279 & 65 & 3684 \\
\hline M-41 & 0,47 & 16,25 & 8,03 & 44,14 & 0,86 & 28,09 & 0,23 & 0,03 & 1,66 & 40 & 78 & 286 & 72 & 1895 \\
\hline M-42 & 0,52 & 3,45 & 7,24 & 45,27 & 1,71 & 39,52 & 0,17 & 0,06 & 1,81 & 47 & 88 & 438 & 76 & 1876 \\
\hline M-44 & 0,70 & 2,16 & 18,08 & 60,26 & 1,00 & 11,15 & 0,98 & 0,04 & 5,18 & 146 & 218 & 50 & 22 & 3446 \\
\hline M-45 & 1,31 & 2,55 & 20,13 & 65,41 & 1,81 & 5,41 & 0,59 & 0,06 & 2,47 & 72 & 209 & 52 & 16 & 1694 \\
\hline M-49 & 0,33 & 2,98 & 13,03 & 50,13 & 1,20 & 28,47 & 0,45 & 0,14 & 3,01 & 56 & 88 & 417 & 222 & 1802 \\
\hline
\end{tabular}

elegidas inicialmente eran las que proporcionaban una mejor separación del conjunto de datos estudiado. Estas ocho variables fueron las siguientes: $\mathrm{Na}_{2} \mathrm{O}, \mathrm{MgO}, \mathrm{Al}_{2} \mathrm{O}_{3}, \mathrm{SiO}_{2}, \mathrm{CaO}$, $\mathrm{Fe}_{2} \mathrm{O}_{3}, \mathrm{Cu}$ y $\mathrm{Zn}$.

Un análisis cluster realizado a partir de las ocho variables mencionadas, utilizando el método del centroide y una matriz de distancias euclídeas, proporcionó un dendrograma en el que la mayoría de las muestras se clasificaban en dos grupos, formados por diferentes niveles de similaridad y compuestos por tres y 11 muestras respectivamente, mientras que tres de las cerámicas aparecían desagrupadas. Una estructura similar de dos grupos y tres muestras desagrupadas, es la que también reflejó el análisis de componentes principales, basado en una matriz de varianza-covarianza a partir de las mismas ocho variables, que se realizó posteriormente con el propósito de contrastar los resultados del análisis cluster. La distinción composicional de estos dos grupos se ilustra en la Figura 6, donde se ofrece una representación de las muestras y su agrupación según su puntuación en los dos primeros componentes, los cuales sumaron el 75,84 \% de la variación total en los datos. Con respecto al primer componente, que explicó un 53,69\% de la variación, las variables $\mathrm{MgO}$ y CaO se correlacionaron de forma positiva, mientras que las variables $\mathrm{Cu}$ y $\mathrm{Al}_{2} \mathrm{O}_{3}$ lo hicieron de manera negativa. En el segundo componente, con un 22,15\% de la variación, fue la variable $\mathrm{CaO}$ la que se correlacionó de forma positiva, mientras que las variables $\mathrm{MgO}, \mathrm{Cu}$ y $\mathrm{Zn}$ se correlacionaron negativamente.

Con el fin de estimar la validez estadística de los grupos obtenidos con los análisis cluster y de componentes principales, se realizó por último un análisis canónico discriminante en el que también se calcularon las distancias de Mahalanobis de cada muestra con respecto a los centroides de cada agrupación (10). Los resultados de este análisis confirmaron las dos agrupaciones establecidas, así como la existencia de tres muestras que no pertenecían a ninguna de ellas. En todos los casos, las muestras presentaron un $100 \%$ de probabilidad de pertenencia a su grupo, excepto las que aparecían

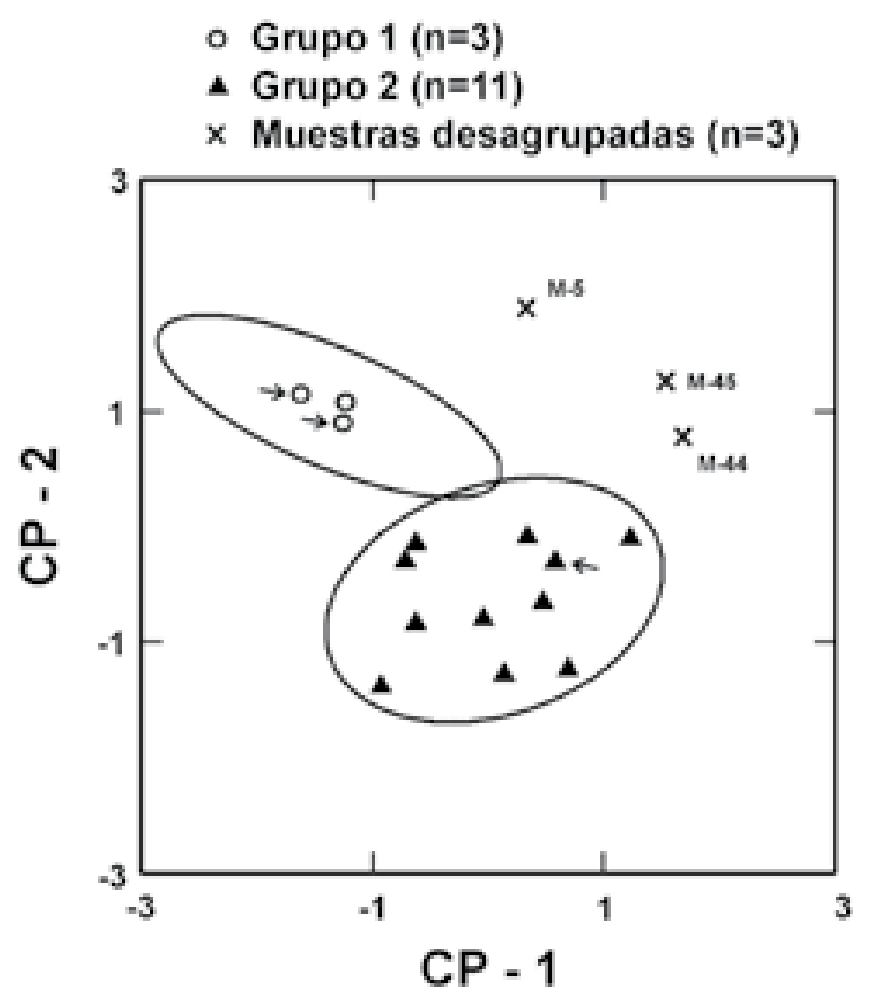

Fig. 6- Representación de las muestras y sus agrupaciones según la puntuación en los dos primeros componentes, a partir de la transformación logarítmica de las concentraciones obtenidas mediante FRX ( $n=17$ muestras), utilizando una matriz de varianza-covarianza. Las elipses representan el $90 \%$ de confidencia de pertenencia a cada grupo. Las flechas señalan la ubicación de las muestras tipificadas como ocarinas. O Grupo $1(\mathrm{n}=3)$. $\Delta$ Grupo $2(\mathrm{n}=11)$. x Muestras desagrupadas $(n=3)$. 
desagrupadas. En cuanto a las distancias de Mahalanobis, el Grupo 1 se mostró muy homogéneo, ya que sus puntuaciones se situaron entre 2,2 y 6,6 respecto a su centroide. Por el contrario, el Grupo 2 presentó una mayor heterogeneidad, con puntuaciones a su centroide que iban desde 3,3 hasta 10,4. En la Tabla III se presentan los valores promedio, las desviaciones estándar y los coeficientes de variación de las dos agrupaciones obtenidas en los análisis estadísticos. y calcio, como puede observarse en el gráfico bivariante de la Figura 7, pertenecería al Grupo 1. Por lo demás, otros elementos que contribuyen a su separación de este grupo, son los óxidos de aluminio, titanio y hierro, cuyas concentraciones son en todos los casos más elevadas.

El Grupo 2 está compuesto por 11 muestras (M-3, M-4, M-7, M-10, M-11, M-12, M-14, M-16, M-17, M-42 y M-49) y se

TABLA III. VALORES PROMEDIO DE LAS DOS AGRUPACIONES OBTENIDAS EN LOS ANÁLISIS ESTADÍSTICOS REALIZADOS A PARTIR DE LOS DATOS DE FRX.

\begin{tabular}{|c|c|c|c|c|c|c|c|c|c|c|c|c|c|c|}
\hline & $\begin{array}{c}\mathrm{Na}_{2} \mathrm{O} \\
(\%)\end{array}$ & $\begin{array}{c}\mathrm{MgO} \\
(\%)\end{array}$ & $\begin{array}{c}\mathrm{Al}_{2} \mathrm{O}_{3} \\
(\%)\end{array}$ & $\begin{array}{l}\mathrm{SiO}_{2} \\
(\%)^{2}\end{array}$ & $\begin{array}{l}\mathrm{K}_{2} \mathrm{O} \\
(\%)\end{array}$ & $\begin{array}{l}\mathrm{CaO} \\
(\%)\end{array}$ & $\begin{array}{l}\mathrm{TiO}_{2} \\
(\%)\end{array}$ & $\begin{array}{c}\mathrm{MnO} \\
(\%)\end{array}$ & $\begin{array}{c}\mathrm{Fe}_{2} \mathrm{O}_{3} \\
(\%)\end{array}$ & $\begin{array}{c}\mathrm{Cu} \\
(\mathrm{ppm})\end{array}$ & $\begin{array}{c}\mathrm{Zn} \\
(\mathrm{ppm})\end{array}$ & $\begin{array}{c}\text { Sr } \\
\text { (ppm) }\end{array}$ & $\begin{array}{c}\mathrm{Zr} \\
(\mathrm{ppm})\end{array}$ & $\begin{array}{c}\text { Ва } \\
\text { (ppm) }\end{array}$ \\
\hline & & & & & & & & & & & & & & \\
\hline \multirow{2}{*}{\multicolumn{15}{|c|}{$\begin{array}{c}\text { Grupo } 1 \\
(\mathrm{n}=3)\end{array}$}} \\
\hline & & & & & & & & & & & & & & \\
\hline Media & 0,39 & 14,05 & 9,31 & 47,70 & 1,15 & 24,96 & 0,27 & 0,04 & 1,96 & 42 & 82 & 344 & 83 & 1201 \\
\hline D.S. & 0,08 & 1,92 & 1,11 & 3,40 & 0,27 & 3,17 & 0,04 & 0,01 & 0,27 & 3 & 9 & 102 & 13 & 703 \\
\hline C.V. & 20,7 & 13,7 & 11,9 & 7,1 & 23,7 & 12,7 & 13,9 & 15,7 & 13,7 & 6,3 & 11,3 & 29,6 & 16,0 & 58,5 \\
\hline & & & & & & & & & & & & & & \\
\hline & & & & & & & & & & & & & & \\
\hline \multirow{2}{*}{\multicolumn{15}{|c|}{$\begin{array}{c}\text { Grupo } 2 \\
(\mathrm{n}=11)\end{array}$}} \\
\hline & & & & & & & & & & & & & & \\
\hline Media & 0,39 & 3,17 & 13,82 & 50,45 & 1,17 & 26,83 & 0,44 & 0,15 & 3,29 & 62 & 103 & 546 & 164 & 1964 \\
\hline D.S. & 0,12 & 1,27 & 5,21 & 3,84 & 0,47 & 8,81 & 0,20 & 0,22 & 1,07 & 33 & 25 & 156 & 68 & 815 \\
\hline C.V. & 30,3 & 40,3 & 37,7 & 7,6 & 40,5 & 32,8 & 45,3 & 142,5 & 32,5 & 53,2 & 24,6 & 28,6 & 41,8 & 41,5 \\
\hline
\end{tabular}

D.S. Desviación estándar $( \pm)$

C.V. Coeficiente de variación $(\%)$

El Grupo 1 está compuesto por sólo tres muestras (M-8, M-9 y M-41) y se caracteriza por la elevada concentración de óxidos de calcio $(24,96 \%)$ y de magnesio $(14,05 \%)$ (Tabla III), que eran las variables que se correlacionaban positivamente con el primer componente en el análisis de componentes principales y que contribuían a separarlo del Grupo 2. Los coeficientes de variación se sitúan, para la mayoría de los elementos, por debajo del $16 \%$, siendo sólo los correspondientes a los óxidos de sodio y potasio, por un lado, y al estroncio y al bario, por otro, los que muestran valores por encima del $20 \%$. Este hecho demuestra que el Grupo 1, a pesar de contener un corto número de muestras, que impide una mejor valoración estadística, es composicionalmente muy homogéneo como ya apuntaban las distancias de Mahalanobis. Este grupo coincide con el que presentaba dolomita como fase predominante en los análisis por DRX y espectroscopía infrarroja, por lo que las altas concentraciones de óxidos de calcio y magnesio deben atribuirse a la presencia de esta fase. No obstante, en los análisis por DRX y FT-IR, la muestra M-5 mostraba aparentemente un mayor contenido de cuarzo. Este aspecto se ve reflejado en su composición química, que arroja una concentración mayor en óxido de silicio $(50,36 \%)$, sin duda relacionado con el contenido de cuarzo, y menor en los óxidos de magnesio $(8,44 \%)$ y calcio $(20,11 \%)$, con respecto a las muestras del Grupo 1 (Tabla III). Por este motivo, en el análisis estadístico la muestra M-5 aparece como desagrupada a pesar de que, atendiendo a su concentración en óxidos de magnesio caracteriza por un mayor contenido en óxido de calcio $(26,83$ $\%$ ) y una menor concentración de óxido de magnesio $(3,17 \%)$, con respecto al Grupo 1 (Tabla III). Asimismo, los contenidos de óxido de aluminio (13,82\%), cobre (62 ppm) y cinc (103 ppm), que eran las variables que se correlacionaban de forma negativa con los dos primeros componentes en el análisis de componentes principales, también contribuyen a separarlo del grupo anterior, puesto que sus concentraciones son mayores. Lo mismo ocurre con los óxidos de silicio $(50,45 \%)$, titanio $(0,44 \%)$ y hierro $(3,29 \%)$. Los coeficientes de variación, aunque por debajo del $10 \%$ para el óxido de silicio y cercanos al $30 \%$ para los óxidos de sodio, calcio y hierro o para los elementos estroncio y cinc (Tabla III), son en general elevados, lo cual demuestra que el grupo es bastante heterogéneo como ya se percibía en los valores correspondientes a las distancias de Mahalanobis. Este grupo coincide con el que presentaba calcita como fase mayoritaria en los análisis por DRX y espectroscopía infrarroja, por lo que las altas concentraciones de óxido de calcio deben atribuirse a la presencia de esta fase. Por otro lado, estos análisis mostraban una mayor presencia de cuarzo con respecto a las muestras de la otra agrupación, lo que se ve reflejado en la mayor concentración de óxido de silicio (50,45 \% en el Grupo 2; 47,70 \% en el Grupo 1) (Tabla III).

Finalmente, las muestras desagrupadas M-44 y M-45, presentan unas claras diferencias composicionales con los Grupos 1 y 2, ya que son las cerámicas con las concentraciones 
más bajas en óxidos de magnesio y calcio y las más elevadas en óxidos de sodio, aluminio, silicio y titanio (Tabla III). Estas dos muestras coinciden con el grupo 3 que presentaba cuarzo como fase predominante en los espectros de infrarrojo, hecho que se corrobora en el análisis químico por FRX al ser las muestras que presentan los mayores contenidos en óxido de silicio (60,26 y 65,41\%, respectivamente) (Tabla III). Las características de sus difractogramas también ratifican el alto contenido en óxido de silico, ya que muestran una banda ancha amorfa que podría corresponderse con sílice vitrea. Estas dos muestras pueden constituir otra agrupación distinta a las dos anteriores, aunque el escaso número de ejemplares representado en este muestreo impide, por el momento, una evaluación estadística más pormenorizada.

\subsection{Interpretación arqueológica}

Los análisis llevados a cabo permiten establecer que las cerámicas estudiadas fueron manufacturadas con tres tipos distintos de materia prima arcillosa. Una materia prima arcillosa rica en carbonatos mixtos de calcio y magnesio, que se relaciona con el grupo de cerámicas con dolomita; otra materia prima rica principalmente en carbonatos de calcio, relacionada con el grupo de cerámicas con calcita; y una tercera sin dolomita, con abundante cuarzo y mucho menos rica en carbonato de calcio.

En términos arqueométricos, la estimación directa y absoluta de la temperatura de cocción de una cerámica arqueológica nunca se puede realizar, ya que no es posible determinar ciertos parámetros como el tipo de atmósfera durante la cocción o la duración de la misma. Por este motivo, se maneja el concepto de temperatura de cocción equivalente, que consiste en una aproximación indirecta a la temperatura de cocción a través de su relación con los cambios mineralógicos y microestructurales que experimenta la materia prima con la que se manufacturó la cerámica. Las estimaciones de temperatura sobre cerámica arqueológica deben valorarse, en cualquier caso, con un criterio flexible puesto que, además, hay que tener en cuenta que en los procesos de cocción realizados con medios tradicionales, pueden darse diferencias de temperatura de más de $100^{\circ} \mathrm{C}$ dentro de una misma estructura (13-16). En los materiales analizados en este trabajo no se han detectado fases de neoformación producidas por la disociación de la calcita o la dolomita por efecto de la temperatura y su posterior reacción con los filosilicatos de la arcilla. Habida cuenta que la descomposición de estos carbonatos comienza en torno a los $750-800{ }^{\circ} \mathrm{C}$ dependiendo del tamaño de partícula y de su mayor o menor cristalinidad (13), el límite superior de temperatura de cocción puede fijarse en este rango de temperaturas. Este dato vendría apoyado también por el hecho de que, en la mayoría de las muestras, todavía se observa la presencia de mica y otros filosilicatos deshidroxilados cuya descomposición total no se produce hasta los $800-850{ }^{\circ} \mathrm{C}$. $(13,16)$. En este contexto, la ausencia de silicatos de calcio, que se forman precisamente en este rango térmico, podría explicarse por el tamaño que presentan los carbonatos (hasta 300-400 $\mu$ m según se observó mediante MO), lo cual retardaría la temperatura de descomposición de los mismos. Por otro lado, tampoco se produjo el crecimiento de fases de óxidos de hierro como la hematita, que se desarrolla a temperaturas superiores a los $700{ }^{\circ} \mathrm{C}$ en atmósfera oxidante (13). Este dato no sólo se explica por la relativamente baja concentración de óxido de hierro en las cerámicas analizadas (Tabla II), sino también porque en entornos ricos en calcio, el hierro no se oxida fácilmente, produciéndose en consecuencia una inhibición del crecimiento de óxidos de hierro, según han establecido Maniatis y col. (17). Es por ello, también, por lo que estas cerámicas, aparte de haber sido cocidas en atmósferas oxidantes, presentan un color ocre predominantemente. Dado que en la mayor parte de los difractogramas las reflexiones correspondientes a los filosilicatos casi han desaparecido (Figura 4), el límite inferior de temperatura de cocción podría fijarse en torno a los $650-700{ }^{\circ} \mathrm{C}$. Ahora bien, las muestras de la tercera agrupación fueron sometidas a una temperatura notoriamente más elevada, probablemente entre los 700 y 800 ${ }^{\circ} \mathrm{C}$, puesto que han perdido prácticamente su cristalinidad, en comparación con las muestras de los Grupos 1 y 2. Por lo tanto, la temperatura de cocción equivalente de las cerámicas aquí analizadas podría situarse entre los $700-800{ }^{\circ} \mathrm{C}$ o quizás algo más baja, lo cual está en consonancia con los datos aportados por otros estudios arqueométricos sobre cerámicas mayas $(18,19)$.

En el Grupo 1 aparecen representadas figurillas procedentes de los niveles 6, 7 y 8, mientras que el Grupo 2 contiene figurillas de todos los niveles representados en el muestreo. Además, las cerámicas de ambos grupos proceden de distintas ubicaciones dentro de la Estructura II (Tabla 1). Estos datos indican que los grupos no responden a una posible concentración microespacial, excepto en el caso de las muestras desagrupadas M-44 y M-45, que proceden únicamente de los cuartos 34-35 en donde no aparecen cerámicas de ninguno

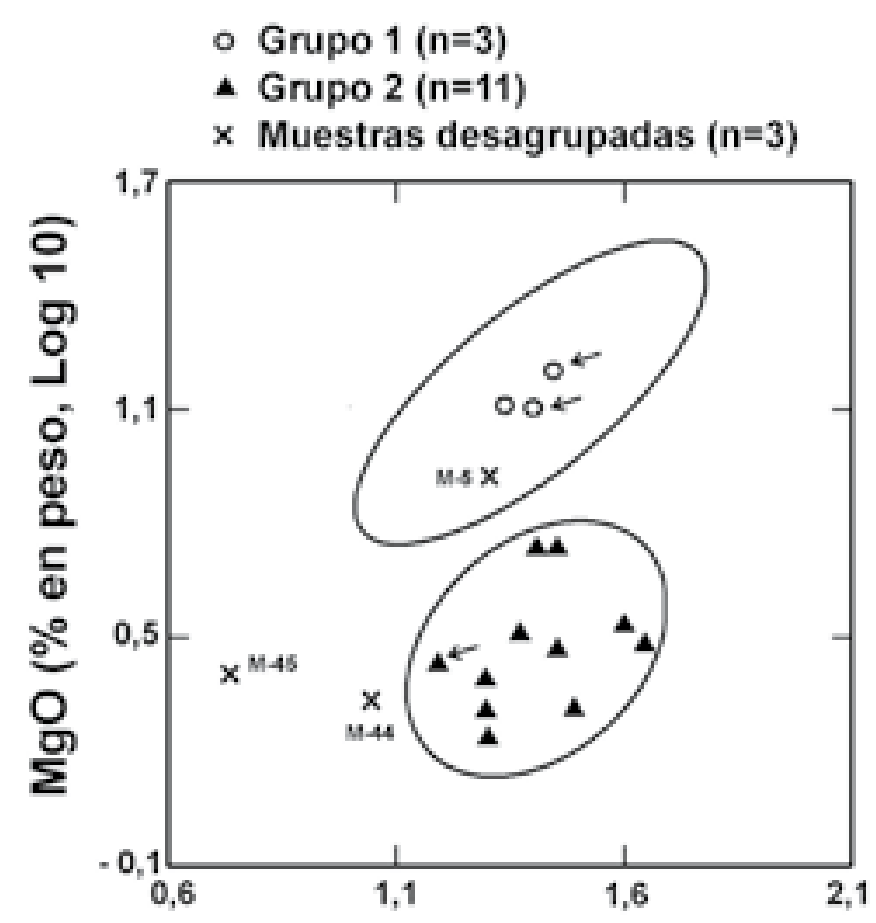

$\mathrm{CaO}(\%$ en peso, $\log 10)$

Fig. 7- Gráfico bivariante realizado a partir de la transformación logarítmica de las concentraciones de óxido de calcio y de óxido de magnesio obtenidas mediante FRX ( $n=17$ muestras), según las agrupaciones del análisis de componentes principales. Las elipses representan el 90 $\%$ de confidencia de pertenencia a cada grupo. Las flechas señalan la ubicación de las muestras tipificadas como ocarinas. OGrupo $1(n=3)$. $\Delta$ Grupo 2 (n=11). x Muestras desagrupadas $(n=3)$. 
de los otros dos grupos identificados. Ahora bien, los datos sí parecen indicar que existió una posible especialización funcional, ya que dos de las tres ocarinas presentes en el muestreo (M-8 y M-41) pertenecen al Grupo 1 (muestras señaladas con flechas en las Figuras 6 y 7). Dicho grupo, que es el único en donde se identificó dolomita, parece que se reservó a piezas con un determinado valor, puesto que la otra muestra de este grupo (M-9) conservaba restos de pigmento azul maya (20). De todos modos, la tercera ocarina analizada (M-16) y la flauta (M-17) se asociaron al Grupo 2, lo que demuestra que no todos los instrumentos musicales se manufacturaban con la materia prima arcillosa rica en dolomita asociada al Grupo 1. Por tanto, la hipótesis de que las cerámicas del Grupo 1 pertenecen a un taller especializado en la producción de determinadas piezas cerámicas, necesitará ser confirmada en estudios posteriores.

Finalmente, con los datos obtenidos en esta investigación, resulta difícil valorar si los grupos cerámicos identificados pueden asociarse a distintos talleres o a un solo taller que utilizara diferentes materias primas en función de las piezas a elaborar. En cuanto a la procedencia geográfica, un reciente estudio sobre otros tipos de cerámica maya y sobre distintas materias primas arcillosas provenientes de los alrededores de Calakmul, ha mostrado que una buena parte de estas arcillas contienen montmorillonita y abundantes inclusiones de cuarzo y calcita (19). Teniendo en cuenta que son precisamente estas fases las que se han identificado en las muestras del Grupo 2 y en las muestras desagrupadas M-44 y M-45, podría apuntarse un origen local para estas cerámicas. Sin embargo, en el mencionado trabajo no se indica que se hayan identificado materias primas arcillosas que contengan carbonatos mixtos del tipo dolomita, lo cual podría sugerir un origen foráneo, o al menos de otra zona de la propia región de Calakmul no muestreada en dicho trabajo, para las muestras asociadas al Grupo 1.

\section{CONCLUSIONES}

El análisis arqueométrico mediante distintas técnicas de caracterización fisicoquímica de un conjunto de figurillas cerámicas mayas del Clásico Tardío (600-900 d.C.), procedentes de la Estructura II de la ciudad de Calakmul (Campeche, México), ha proporcionado unos datos de extraordinario interés sobre su tecnología de producción y sobre su posible procedencia geográfica.

Los resultados indican que la mayoría de las figurillas cerámicas (Grupo 2) se manufacturaron con una materia prima arcillosa rica en carbonato de calcio (calcita) que fue cocida, en atmósfera predominantemente oxidante, en torno a los 700$800{ }^{\circ} \mathrm{C}$ o quizás algo más baja. La presencia de las fases cuarzo, calcita y posiblemente montmorillonita sugiere un origen local para estas cerámicas. Junto a éstas, se ha identificado un pequeño grupo de cuatro muestras (Grupo 1) caracterizado por la presencia de carbonatos mixtos de calcio y magnesio (dolomita), que podría ser foráneo o procedente de otra zona de la propia región de Calakmul. A este grupo se asocian dos de los instrumentos musicales tipificados como ocarinas y una muestra con restos de pigmento azul maya, por lo que habría que relacionarlo con una producción especializada en piezas con un determinado valor. La determinación de, al menos, dos producciones distintas elaboradas con materias primas diferentes (una rica en calcita y otra en dolomita), sugiere que la producción de figurillas cerámicas podría estar asociada a distintos talleres o a un solo taller, que utilizara diferentes materias primas en función de las piezas a elaborar, cuya existencia estaría ligada al monopolio que el poder centralizado de la ciudad de Calakmul ejerció sobre la producción de bienes cerámicos en este período.

Por último, la investigación arqueométrica llevada a cabo en este trabajo confirma que la aplicación de técnicas de caracterización fisicoquímica en el estudio de materiales cerámicos arqueológicos proporciona una información muy valiosa sobre la composición, tecnología y procedencia de estos materiales, generando nuevas hipótesis de trabajo y un conocimiento más detallado de la organización socioeconómica y política desarrollada en el estado regional de Calakmul entre el Preclásico Tardío y el Clásico Tardío.

\section{AGRADECIMIENTOS}

Los autores agradecen al Instituto Nacional de Antropología e Historia (INAH, México) la autorización para realizar análisis en las distintas piezas cerámicas. Asimismo, agradecen el apoyo institucional de la Red Temática del CSIC de Patrimonio Histórico y Cultural. Los autores también quieren dar las gracias a Miguel A. Rogerio, IRNAS-CSIC, por la ayuda prestada en la presentación de los datos de la Tabla I. Este trabajo ha sido realizado con cargo al proyecto MCYT MAT2002-02549, financiado en parte con fondos FEDER.

\section{REFERENCIAS BIBLIOGRÁFICAS}

1. R. Carrasco, Calakmul, Campeche, Arqueología Mexicana 3, 46-51, 1996.

2. C. Vidal y M. Domínguez. Eds. Calakmul. Antología. Instituto Nacional de Antropología e Historia (INAH), Campeche, México, 2003.

3. O. Rodríguez Campero, La Gran Plaza de Calakmul, Arqueología Mexicana 7, 22-27, 2000.

4. R. Ruiz Guzmán. Las figurillas e instrumentos musicales de Calakmul, Campeche. Descripción, análisis e interpretación: una tentativa tipológica. Tesis de Licenciatura, Instituto Nacional de Antropología e Historia (INAH), México, 1998.

5. R. Ruiz Guzmán, R.L. Bishop y W.L. Folan. Las figurillas de Calakmul, Campeche: su uso funcional y clasificación sociocultural y química. Centro de Investigaciones Históricas y Sociales, Dr. Román Piña Chan, Universidad Autónoma de Campeche, Campeche, México, 1999.

6. A.J. López, G. Nicolás, M.P. Mateo, V. Piñón, A. Ramil y A. Yáñez, Análisis de cerámicas romanas Terra Sigillata mediante espectroscopía de plasmas inducidos por láser (LIPS), Bol. Soc. Esp. Ceram. V., 44, 373-378, 2005.

7. N. Carmona, M. García-Heras, C. Gil y M.A. Villegas, Vidrios y grisallas del s. XV de la Cartuja de Miraflores (Burgos): caracterización y estado de conservación, Bol. Soc. Esp. Ceram. V., 44, 251-258, 2005.

8. E.J. Roca, A. Ruiz-Conde y P.J. Sánchez-Soto, Preparación de secciones estratigráficas: aspectos prácticos del análisis de estratos en obras del Patrimonio Cultural (pigmentos y soportes), Bol. Soc. Esp. Ceram. V., 44, 382-386, 2005

9. G.E. De Benedetto, R. Laviano, L. Sabbatini y P.G. Zambonin, Infrared spectroscopy in the mineralogical characterization of ancient pottery, J. Cult. Herit. 3, 177-186, 2002.

10. M.J. Baxter. Exploratory multivariate analysis in archaeology. Edinburgh University Press, Edinburgh, 1994.

11. R.L. Bishop y H. Neff, Compositional data analysis in archaeology, pp. 5786 en Archaeological Chemistry IV. Ed. R.O. Allen, Advances in Chemistry Series 220, The American Chemical Society, Washington D.C., 1989.

12. H. Bearat y D. Dufournier, Quelques expériences sur la fixation du phosphore par les céramiques, Revue d'Archéométrie 18, 65-73, 1994.

13. P.M. Rice. Pottery Analysis: a Sourcebook. Chicago University Press, Chicago, 1987.

14. M. García Heras, Estudio arqueométrico de la cerámica de Izana (Soria) y de otros yacimientos celtibéricos del Alto Duero, Bol. Soc. Esp. Ceram. V., 33, 315-325, 1994.

15. M.S. Tite, Firing temperature determinations. How and why?, pp. 37-42 en The Aims of Laboratory Analyses of Ceramics in Archaeology. Eds. A. Lindahl y O. Stilborg, Historie och Antikvitets Akademien, Konferenser 34, Estocolmo, 1995. 
16. A. García Verduch. "Algunos conceptos básicos de la cocción rápida”. Bol. Soc. Esp. Ceram. V., 23, 387-392, 1984.

17. Y. Maniatis, A. Simopoulos y A. Kostikas, Mössbauer study of the effect of calcium content on iron oxide transformations in fired clays, J. Am. Ceram. Soc. 64, 263-269, 1981.

18. P.M. Rice, Maya pottery techniques and technology, pp. 113-132 en Ancient Technology to Modern Science. Ed. W.D. Kingery, Ceramics and Civilization Vol. 1, American Ceramic Society, Columbus (Ohio), 1985.
19. M.R. Domínguez, M.E. Espinosa, V. Rodríguez y W.J. Folan, Resultado de los análisis por MEB y DRX de cerámica arqueológica de Calakmul, Campeche, pp. 543-553 en XV Simposio de Investigaciones Arqueológicas en Guatemala. Eds. J.P. Laporte, H. Escobedo y B. Arroyo, Museo Nacional de Arqueología y Etnología, Guatemala, 2002.

20. M.J. Yacamán, L. Rendón, J. Arenas y M.C. Serra. Maya blue paint: an ancient nanostructured material, Science 273, 223, 1996.

Recibido: $\quad 11.10 .05$

Aceptado: 03.04 .06 